\title{
CLASSES WITH NEGATIVE COEFFICIENTS AND STARLIKE WITH RESPECT TO OTHER POINTS II
}

\author{
SUZEINI ABDUL HALIM, AINI JANTENG AND MASLINA DARUS
}

\begin{abstract}
A class $S_{s}^{\star} T(\alpha, \beta, \sigma, k)$ of functions $f$ which are analytic and univalent in the open unit disk $D=\{z:|z|<1\}$ given by $f(z)=z-\sum_{n=2}^{\infty} a_{n} z^{n}$ and satisfying the condition

$$
\left|\frac{z f^{\prime}(z)}{f(z)-f(-z)}-k\right|<\beta\left|\frac{\alpha z f^{\prime}(z)}{f(z)-f(-z)}-(2 \sigma-k)\right|
$$

for $0 \leq \alpha \leq 1,0<\beta \leq 1,0 \leq \sigma \leq \frac{1}{2}<k \leq 1, z \in D$, is introduced and studied. An analogous class $S_{c}^{\star} T(\alpha, \beta, \sigma, k)$ and $S_{s c}^{\star} T(\alpha, \beta, \sigma, k)$ are also examined.
\end{abstract}

\section{Introduction}

Let $\mathcal{S}$ be the class of functions $f$ which are analytic and univalent in the open unit disk $D=\{z:|z|<1\}$ given by

$$
f(z)=z+\sum_{n=2}^{\infty} a_{n} z^{n}
$$

and $a_{n}$ a complex number. Let $S_{s}^{\star}$ be the subclass of $\mathcal{S}$ consisting of functions given by (1) satisfying

$$
R e\left\{\frac{z f^{\prime}(z)}{f(z)-f(-z)}\right\}>0, \quad z \in D
$$

These functions are called starlike with respect to symmetric points and were introduced by Sakaguchi [4]. El-Ashwa and Thomas [2] obtained various results concerning functions in $S_{s}^{\star}$ and two other classes namely the class $S_{c}^{\star}$ of functions starlike with respect to conjugate points and the class $S_{s c}^{\star}$ of functions starlike with respect to symmetric conjugate points.

Now, we denote $\mathcal{T}$ the class consisting of functions $f$ of the form

$$
f(z)=z-\sum_{n=2}^{\infty} a_{n} z^{n}
$$

Received April 25, 2005.

2000 Mathematics Subject Classification. Primary 30C45.

Key words and phrases. Analytic, univalent, starlike with respect to symmetric points. 
where $a_{n}$ is a non negative real number.

For $f \in \mathcal{T}$, Halim et al. [3] studied the class $S_{s}^{\star} T(\alpha, \beta), 0 \leq \alpha \leq 1, \frac{1}{2}<\beta \leq$ 1 , consisting of functions $f \in \mathcal{T}$ and starlike with respect to symmetric points. An analogous results are also obtained for the class $S_{c}^{\star} T(\alpha, \beta), 0 \leq \alpha \leq 1, \frac{1}{2}<\beta \leq 1$, consisting of functions $f \in \mathcal{T}$ and starlike with respect to conjugate points and the class $S_{s c}^{\star} T(\alpha, \beta), 0 \leq \alpha \leq 1, \frac{1}{2}<\beta \leq 1$, consisting of functions $f \in \mathcal{T}$ and starlike with respect to symmetric conjugate points.

For this paper, we consider a class $S_{s}^{\star} T(\alpha, \beta, \sigma, k), 0 \leq \alpha \leq 1,0<\beta \leq 1,0 \leq \sigma \leq$ $\frac{1}{2}<k \leq 1$, consisting of functions $f \in \mathcal{T}$ and starlike with respect to symmetric points as follows:

Definition 1. A function $f \in S_{s}^{\star} T(\alpha, \beta, \sigma, k)$ if it satisfies

$$
\left|\frac{z f^{\prime}(z)}{f(z)-f(-z)}-k\right|<\beta\left|\frac{\alpha z f^{\prime}(z)}{f(z)-f(-z)}-(2 \sigma-k)\right|
$$

for some $0 \leq \alpha \leq 1,0<\beta \leq 1,0 \leq \sigma \leq \frac{1}{2}<k \leq 1$ and $z \in D$.

An analogous results are also obtained for the class of functions $f \in \mathcal{T}$ and starlike with respect to conjugate points and functions starlike with respect to symmetric conjugate points. These classes are defined as below:

Definition 2. A function $f \in S_{c}^{\star} T(\alpha, \beta, \sigma, k)$ if it satisfies

$$
\left|\frac{z f^{\prime}(z)}{f(z)+\overline{f(\bar{z})}}-k\right|<\beta\left|\frac{\alpha z f^{\prime}(z)}{f(z)+\overline{f(\bar{z})}}-(2 \sigma-k)\right|
$$

for some $0 \leq \alpha \leq 1,0<\beta \leq 1,0 \leq \sigma \leq \frac{1}{2}<k \leq 1$ and $z \in D$.

Definition 3. A function $f \in S_{s c}^{\star} T(\alpha, \beta, \sigma, k)$ if it satisfies

$$
\left|\frac{z f^{\prime}(z)}{f(z)-\overline{f(-\bar{z})}}-k\right|<\beta\left|\frac{\alpha z f^{\prime}(z)}{f(z)-\overline{f(-\bar{z})}}-(2 \sigma-k)\right|
$$

for some $0 \leq \alpha \leq 1,0<\beta \leq 1,0 \leq \sigma \leq \frac{1}{2}<k \leq 1$ and $z \in D$.

\section{Coefficient Inequalities}

We first state some preliminary lemmas, required for proving our result.

Lemma 1.([5]) If $f \in \mathcal{T}$ then $\sum_{n=2}^{\infty} n\left|a_{n}\right|<1$.

Lemma 2. If $f \in \mathcal{T}$ then $\sum_{n=2}^{\infty}\left(n \alpha+(k-2 \sigma)\left(1-(-1)^{n}\right)\right)\left|a_{n}\right|<\alpha+2(k-2 \sigma)$. 
Proof. We note that

$$
\begin{aligned}
& \sum_{n=2}^{\infty}\left(n \alpha+(k-2 \sigma)\left(1-(-1)^{n}\right)\right)\left|a_{n}\right| \\
= & \sum_{n=2}^{\infty} n \alpha\left|a_{n}\right|+\sum_{n=2}^{\infty}(k-2 \sigma)\left(1-(-1)^{n}\right)\left|a_{n}\right| \\
= & \alpha \sum_{n=2}^{\infty} n\left|a_{n}\right|+(k-2 \sigma) \sum_{n=2}^{\infty}\left(1-(-1)^{n}\right)\left|a_{n}\right| \\
< & \alpha(1)+(k-2 \sigma) 2(1)=\alpha+2(k-2 \sigma), \quad \text { by (Lemma 1), }
\end{aligned}
$$

as required.

Lemma 3. If $f \in \mathcal{T}$ then $\sum_{n=2}^{\infty}(n \alpha+2(k-2 \sigma))\left|a_{n}\right|<\alpha+2(k-2 \sigma)$.

Proof. We note that

$$
\begin{aligned}
& \sum_{n=2}^{\infty}(n \alpha+2(k-2 \sigma))\left|a_{n}\right| \\
= & \sum_{n=2}^{\infty} n \alpha\left|a_{n}\right|+\sum_{n=2}^{\infty} 2(k-2 \sigma)\left|a_{n}\right| \\
= & \alpha \sum_{n=2}^{\infty} n\left|a_{n}\right|+2(k-2 \sigma) \sum_{n=2}^{\infty}\left|a_{n}\right| \\
< & \alpha(1)+2(k-2 \sigma)(1)=\alpha+2(k-2 \sigma), \text { by (Lemma 1), }
\end{aligned}
$$

as required.

For $S_{s}^{\star} T(\alpha, \beta, \sigma, k)$, we have the following:

Theorem 1. Let $f \in \mathcal{T}$. A function $f \in S_{s}^{\star} T(\alpha, \beta, \sigma, k)$ if and only if

$$
\sum_{n=2}^{\infty}\left(\frac{(1+\beta \alpha) n}{\beta(2(k-2 \sigma)+\alpha)-(2 k-1)}+\frac{\beta(k-2 \sigma)\left(1-(-1)^{n}\right)-k\left(1-(-1)^{n}\right)}{\beta(2(k-2 \sigma)+\alpha)-(2 k-1)}\right) a_{n} \leq 1
$$

for $0 \leq \alpha \leq 1, \frac{1}{2}<\beta \leq 1$ and $0 \leq \sigma \leq \frac{1}{2}<k \leq 1$.

Proof. First we prove the 'if' part. We adopt the method used by Clunie and Keogh 
[1]. We write

$$
\begin{aligned}
& \left|z f^{\prime}(z)-k(f(z)-f(-z))\right|-\beta\left|\alpha z f^{\prime}(z)-(2 \sigma-k)(f(z)-f(-z))\right| \\
= & \left|(1-2 k) z-\sum_{n=2}^{\infty}\left(n-k\left(1-(-1)^{n}\right)\right) a_{n} z^{n}\right|-\beta \mid(2(k-2 \sigma)+\alpha) z \\
& -\sum_{n=2}^{\infty}\left(n \alpha+(k-2 \sigma)\left(1-(-1)^{n}\right)\right) a_{n} z^{n} \mid \\
\leq & \sum_{n=2}^{\infty}\left(n-k\left(1-(-1)^{n}\right)\right)\left|a_{n}\right| r^{n}+(2 k-1) r-\beta(2(k-2 \sigma)+\alpha) r \\
& +\sum_{n=2}^{\infty} \beta\left(n \alpha+(k-2 \sigma)\left(1-(-1)^{n}\right)\right)\left|a_{n}\right| r^{n} \\
< & {\left[\sum_{n=2}^{\infty}\left(n-k\left(1-(-1)^{n}\right)\right)\left|a_{n}\right|+(2 k-1)-\beta(2(k-2 \sigma)+\alpha)\right.} \\
& \left.+\sum_{n=2}^{\infty} \beta\left(n \alpha+(k-2 \sigma)\left(1-(-1)^{n}\right)\right)\left|a_{n}\right|\right] r \\
= & {\left[\sum_{n=2}^{\infty}\left((1+\beta \alpha) n+\beta(k-2 \sigma)\left(1-(-1)^{n}\right)-k\left(1-(-1)^{n}\right)\right) a_{n}\right.} \\
& -(\beta(2(k-2 \sigma)+\alpha)-(2 k-1))] r \\
\leq & 0 \text { by }(3) .
\end{aligned}
$$

Thus,

$$
\left|\frac{\frac{z f^{\prime}(z)}{f(z)-f(-z)}-k}{\frac{\alpha z f^{\prime}(z)}{f(z)-f(-z)}-(2 \sigma-k)}\right|<\beta
$$

and hence $f \in S_{s}^{\star} T(\alpha, \beta, \sigma, k)$.

To prove the 'only if' part, we write

$$
\begin{aligned}
& \left|\frac{\frac{z f^{\prime}(z)}{f(z)-f(-z)}-k}{\frac{\alpha z f^{\prime}(z)}{f(z)-f(-z)}-(2 \sigma-k)}\right| \\
= & \left|\frac{(1-2 k)-\sum_{n=2}^{\infty}\left(n-k\left(1-(-1)^{n}\right)\right) a_{n} z^{n-1}}{2(k-2 \sigma)+\alpha-\sum_{n=2}^{\infty}\left(n \alpha+(k-2 \sigma)\left(1-(-1)^{n}\right)\right) a_{n} z^{n-1}}\right|<\beta .
\end{aligned}
$$

Since $f$ is analytic, continuous and non constant in $\mathcal{D}$, the maximum modulus principle 
states that

$$
\begin{aligned}
& \left|\frac{(1-2 k)-\sum_{n=2}^{\infty}\left(n-k\left(1-(-1)^{n}\right)\right) a_{n} z^{n-1}}{2(k-2 \sigma)+\alpha-\sum_{n=2}^{\infty}\left(n \alpha+(k-2 \sigma)\left(1-(-1)^{n}\right)\right) a_{n} z^{n-1}}\right| \\
= & \left|\frac{(2 k-1)+\sum_{n=2}^{\infty}\left(n-k\left(1-(-1)^{n}\right)\right) a_{n} z^{n-1}}{2(k-2 \sigma)+\alpha-\sum_{n=2}^{\infty}\left(n \alpha+(k-2 \sigma)\left(1-(-1)^{n}\right)\right) a_{n} z^{n-1}}\right| \\
\leq & \frac{(2 k-1)+\sum_{n=2}^{\infty}\left(n-k\left(1-(-1)^{n}\right)\right)\left|a_{n}\right||z|^{n-1}}{2(k-2 \sigma)+\alpha-\sum_{n=2}^{\infty}\left(n \alpha+(k-2 \sigma)\left(1-(-1)^{n}\right)\right)\left|a_{n}\right||z|^{n-1}}, \quad \text { by (Lemma 2) } \\
< & \frac{(2 k-1)+\sum_{n=2}^{\infty}\left(n-k\left(1-(-1)^{n}\right)\right) a_{n} r^{n-1}}{2(k-2 \sigma)+\alpha-\sum_{n=2}^{\infty}\left(n \alpha+(k-2 \sigma)\left(1-(-1)^{n}\right)\right) a_{n} r^{n-1}}=f(r) .
\end{aligned}
$$

Since $f \in S_{s}^{\star} T(\alpha, \beta, \sigma, k)$ and $|z|<r<1$, we obtain

$$
\left\{\frac{(2 k-1)+\sum_{n=2}^{\infty}\left(n-k\left(1-(-1)^{n}\right)\right) a_{n} r^{n-1}}{2(k-2 \sigma)+\alpha-\sum_{n=2}^{\infty}\left(n \alpha+(k-2 \sigma)\left(1-(-1)^{n}\right)\right) a_{n} r^{n-1}}\right\}<\beta
$$

for any $z \in \mathcal{D}$. Now letting $r \rightarrow 1$ in (4), we obtain

$$
\begin{aligned}
& (2 k-1)+\sum_{n=2}^{\infty}\left(n-k\left(1-(-1)^{n}\right)\right) a_{n} \\
\leq & \beta\left(2(k-2 \sigma)+\alpha-\sum_{n=2}^{\infty}\left(n \alpha+(k-2 \sigma)\left(1-(-1)^{n}\right)\right) a_{n}\right)
\end{aligned}
$$

and hence, we obtain

$$
\sum_{n=2}^{\infty}\left(\frac{(1+\beta \alpha) n}{\beta(2(k-2 \sigma)+\alpha)-(2 k-1)}+\frac{\beta(k-2 \sigma)\left(1-(-1)^{n}\right)-k\left(1-(-1)^{n}\right)}{\beta(2(k-2 \sigma)+\alpha)-(2 k-1)}\right) a_{n} \leq 1
$$

as required. This completes the proof of the theorem.

Remark 1. We note that the case $0<\beta \leq \frac{1}{2}$ remains open.

The result in Theorem 1 is sharp for functions given by

$$
f_{n}(z)=z-\frac{\beta(2(k-2 \sigma)+\alpha)-(2 k-1)}{(1+\beta \alpha) n+\beta(k-2 \sigma)\left(1-(-1)^{n}\right)-k\left(1-(-1)^{n}\right)} z^{n}, \quad n \geq 2 .
$$

Corollary 1. If $f \in S_{s}^{\star} T(\alpha, \beta, \sigma, k)$ then

$$
a_{n} \leq \frac{\beta(2(k-2 \sigma)+\alpha)-(2 k-1)}{(1+\beta \alpha) n+\beta(k-2 \sigma)\left(1-(-1)^{n}\right)-k\left(1-(-1)^{n}\right)}, \quad n \geq 2 .
$$

Next, similar coefficient properties for functions which belong to $S_{c}^{\star} T(\alpha, \beta, \sigma, k)$ and $S_{s c}^{\star} T(\alpha, \beta, \sigma, k)$ are obtained. Similar method of proving is used for Theorem 2 and Theorem 3 . 
Theorem 2. Let $f \in \mathcal{T}$. A function $f \in S_{c}^{\star} T(\alpha, \beta, \sigma, k)$ if and only if

$$
\sum_{n=2}^{\infty}\left(\frac{(1+\beta \alpha) n}{\beta(2(k-2 \sigma)+\alpha)-(2 k-1)}+\frac{2(\beta(k-2 \sigma)-k)}{\beta(2(k-2 \sigma)+\alpha)-(2 k-1)}\right) a_{n} \leq 1
$$

for $0 \leq \alpha \leq 1, \frac{1}{2}<\beta \leq 1$ and $0 \leq \sigma \leq \frac{1}{2}<k \leq 1$.

Proof. First we prove the 'if' part. We write

$$
\begin{aligned}
& \left|z f^{\prime}(z)-k(f(z)+\overline{f(\bar{z})})\right|-\beta\left|\alpha z f^{\prime}(z)-(2 \sigma-k)(f(z)+\overline{f(\bar{z})})\right| \\
= & \left|(1-2 k) z-\sum_{n=2}^{\infty}(n-2 k) a_{n} z^{n}\right|-\beta\left|(2(k-2 \sigma)+\alpha) z-\sum_{n=2}^{\infty}(n \alpha+2(k-2 \sigma)) a_{n} z^{n}\right| \\
\leq & \sum_{n=2}^{\infty}(n-2 k)\left|a_{n}\right| r^{n}+(2 k-1) r-\beta(2(k-2 \sigma)+\alpha) r+\sum_{n=2}^{\infty} \beta(n \alpha+2(k-2 \sigma))\left|a_{n}\right| r^{n} \\
< & {\left[\sum_{n=2}^{\infty}(n-2 k)\left|a_{n}\right|+(2 k-1)-\beta(2(k-2 \sigma)+\alpha)+\sum_{n=2}^{\infty} \beta(n \alpha+2(k-2 \sigma))\left|a_{n}\right|\right] r } \\
= & {\left[\sum_{n=2}^{\infty}((1+\beta \alpha) n+2(\beta(k-2 \sigma)-k)) a_{n}-(\beta(2(k-2 \sigma)+\alpha)-(2 k-1))\right] r } \\
\leq & 0 \quad \text { by }(5) .
\end{aligned}
$$

Thus,

and hence $f \in S_{s}^{\star} T(\alpha, \beta, \sigma, k)$.

$$
\left|\frac{\frac{z f^{\prime}(z)}{f(z)+\overline{f(\bar{z})}}-k}{\frac{\alpha z f^{\prime}(z)}{f(z)+\overline{f(\bar{z})}}-(2 \sigma-k)}\right|<\beta
$$

To prove the 'only if' part, as before we write

$$
\left|\frac{\frac{z f^{\prime}(z)}{f(z)+\overline{f(\bar{z})}}-k}{\frac{\alpha z f^{\prime}(z)}{f(z)+\overline{f(\bar{z})}}-(2 \sigma-k)}\right|=\left|\frac{(1-2 k)-\sum_{n=2}^{\infty}(n-2 k) a_{n} z^{n-1}}{2(k-2 \sigma)+\alpha-\sum_{n=2}^{\infty}(n \alpha+2(k-2 \sigma)) a_{n} z^{n-1}}\right|<\beta .
$$

Since $f$ is analytic, continuous and non constant in $\mathcal{D}$, the maximum modulus principle states that

$$
\begin{aligned}
& \left|\frac{(1-2 k)-\sum_{n=2}^{\infty}(n-2 k) a_{n} z^{n-1}}{2(k-2 \sigma)+\alpha-\sum_{n=2}^{\infty}(n \alpha+2(k-2 \sigma)) a_{n} z^{n-1}}\right| \\
= & \left|\frac{(2 k-1)+\sum_{n=2}^{\infty}(n-2 k) a_{n} z^{n-1}}{2(k-2 \sigma)+\alpha-\sum_{n=2}^{\infty}(n \alpha+2(k-2 \sigma)) a_{n} z^{n-1}}\right| \\
\leq & \frac{(2 k-1)+\sum_{n=2}^{\infty}(n-2 k)\left|a_{n}\right||z|^{n-1}}{2(k-2 \sigma)+\alpha-\sum_{n=2}^{\infty}(n \alpha+2(k-2 \sigma))\left|a_{n}\right||z|^{n-1}}, \quad \text { by (Lemma 3) } \\
< & \frac{(2 k-1)+\sum_{n=2}^{\infty}(n-2 k) a_{n} r^{n-1}}{2(k-2 \sigma)+\alpha-\sum_{n=2}^{\infty}(n \alpha+2(k-2 \sigma)) a_{n} r^{n-1}}=f(r) .
\end{aligned}
$$


Since $f \in S_{c}^{\star} T(\alpha, \beta, \sigma, k)$ and $|z|<r<1$, we obtain

$$
\left\{\frac{(2 k-1)+\sum_{n=2}^{\infty}(n-2 k) a_{n} r^{n-1}}{2(k-2 \sigma)+\alpha-\sum_{n=2}^{\infty}(n \alpha+2(k-2 \sigma)) a_{n} r^{n-1}}\right\}<\beta
$$

for any $z \in \mathcal{D}$. Now letting $r \rightarrow 1$ in $(6)$, we obtain

$$
(2 k-1)+\sum_{n=2}^{\infty}(n-2 k) a_{n} \leq \beta\left(2(k-2 \sigma)+\alpha-\sum_{n=2}^{\infty}(n \alpha+2(k-2 \sigma)) a_{n}\right)
$$

and hence, we obtain

$$
\sum_{n=2}^{\infty}\left(\frac{(1+\beta \alpha) n}{\beta(2(k-2 \sigma)+\alpha)-(2 k-1)}+\frac{2(\beta(k-2 \sigma)-k)}{\beta(2(k-2 \sigma)+\alpha)-(2 k-1)}\right) a_{n} \leq 1
$$

as required. This completes the proof of the theorem.

The result in Theorem 2 is sharp for function $f_{n}$ given by

$$
f_{n}(z)=z-\frac{\beta(2(k-2 \sigma)+\alpha)-(2 k-1)}{(1+\beta \alpha) n+2(\beta(k-2 \sigma)-k)} z^{n} .
$$

Corollary 2. If $f \in S_{c}^{\star} T(\alpha, \beta, \sigma, k)$ then

$$
a_{n} \leq \frac{\beta(2(k-2 \sigma)+\alpha)-(2 k-1)}{(1+\beta \alpha) n+2(\beta(k-2 \sigma)-k)}, \quad n \geq 2 .
$$

For completeness, we state the following result with regards to the class $S_{s c}^{\star} T(\alpha, \beta$, $\sigma, k)$.

Theorem 3. Let $f \in \mathcal{T}$. A function $f \in S_{s c}^{\star} T(\alpha, \beta, \sigma, k)$ if and only if

$$
\sum_{n=2}^{\infty}\left(\frac{(1+\beta \alpha) n}{\beta(2(k-2 \sigma)+\alpha)-(2 k-1)}+\frac{\beta(k-2 \sigma)\left(1-(-1)^{n}\right)-k\left(1-(-1)^{n}\right)}{\beta(2(k-2 \sigma)+\alpha)-(2 k-1)}\right) a_{n} \leq 1
$$

for $0 \leq \alpha \leq 1, \frac{1}{2}<\beta \leq 1$ and $0 \leq \sigma \leq \frac{1}{2}<k \leq 1$.

The result in Theorem 3 is sharp for functions given by

$$
f_{n}(z)=z-\frac{\beta(2(k-2 \sigma)+\alpha)-(2 k-1)}{(1+\beta \alpha) n+\beta(k-2 \sigma)\left(1-(-1)^{n}\right)-k\left(1-(-1)^{n}\right)} z^{n}, \quad n \geq 2 .
$$

Corollary 3. If $f \in S_{s c}^{\star} T(\alpha, \beta, \sigma, k)$ then

$$
a_{n} \leq \frac{\beta(2(k-2 \sigma)+\alpha)-(2 k-1)}{(1+\beta \alpha) n+\beta(k-2 \sigma)\left(1-(-1)^{n}\right)-k\left(1-(-1)^{n}\right)}, \quad n \geq 2 .
$$




\section{Growth Theorem}

A growth property for functions in the class $S_{s}^{\star} T(\alpha, \beta, \sigma, k)$ is given as follows.

Theorem 4. Let the functions $f$ be defined by (2) and belongs to the class $S_{s}^{\star} T(\alpha, \beta$, $\sigma, k)$. Then for $\{z: 0<|z|=r<1\}$,

$$
r-\frac{\beta(2(k-2 \sigma)+\alpha)-(2 k-1)}{2(1+\beta \alpha)} r^{2} \leq|f(z)| \leq r+\frac{\beta(2(k-2 \sigma)+\alpha)-(2 k-1)}{2(1+\beta \alpha)} r^{2} .
$$

The result is sharp.

Proof. First, it is obvious that

$$
\begin{aligned}
& \frac{2(1+\beta \alpha)}{\beta(2(k-2 \sigma)+\alpha)-(2 k-1)} \sum_{n=2}^{\infty} a_{n} \\
\leq & \sum_{n=2}^{\infty}\left(\frac{(1+\beta \alpha) n}{\beta(2(k-2 \sigma)+\alpha)-(2 k-1)}+\frac{\beta(k-2 \sigma)\left(1-(-1)^{n}\right)-k\left(1-(-1)^{n}\right)}{\beta(2(k-2 \sigma)+\alpha)-(2 k-1)}\right) a_{n},
\end{aligned}
$$

and as $f \in S_{s}^{\star} T(\alpha, \beta, \sigma, k)$, using the inequality in Theorem 1 yields

$$
\sum_{n=2}^{\infty} a_{n} \leq \frac{\beta(2(k-2 \sigma)+\alpha)-(2 k-1)}{2(1+\beta \alpha)} .
$$

From (2) with $|z|=r(r<1)$, we have

$$
|f(z)| \leq r+\sum_{n=2}^{\infty} a_{n} r^{n} \leq r+\sum_{n=2}^{\infty} a_{n} r^{2}
$$

and

$$
|f(z)| \geq r-\sum_{n=2}^{\infty} a_{n} r^{n} \geq r-\sum_{n=2}^{\infty} a_{n} r^{2} .
$$

Finally, using (8) in the above inequalities, give the result in Theorem 4.

The result in Theorem 4 is sharp for functions given by

$$
f_{2}(z)=z-\frac{\beta(2(k-2 \sigma)+\alpha)-(2 k-1)}{2(1+\beta \alpha)} z^{2}
$$

at $z= \pm r$.

Next, similar growth results for functions which belong to $S_{c}^{\star} T(\alpha, \beta, \sigma, k)$ and $S_{s c}^{\star} T(\alpha$, $\beta, \sigma, k)$ are obtained. Similar method of proving is used for Theorem 5 and Theorem 6.

Theorem 5. Let the functions $f$ be defined by (2) and belongs to the class $S_{c}^{\star} T(\alpha, \beta$, $\sigma, k)$. Then for $\{z: 0<|z|=r<1\}$,

$$
r-\frac{\beta(2(k-2 \sigma)+\alpha)-(2 k-1)}{2(1+\beta \alpha)+2(\beta(k-2 \sigma)-k)} r^{2} \leq|f(z)| \leq r+\frac{\beta(2(k-2 \sigma)+\alpha)-(2 k-1)}{2(1+\beta \alpha)+2(\beta(k-2 \sigma)-k)} r^{2} .
$$


The result is sharp.

Proof. First, it is obvious that

$$
\begin{aligned}
& \frac{2(1+\beta \alpha)+2(\beta(k-2 \sigma)-k)}{\beta(2(k-2 \sigma)+\alpha)-(2 k-1)} \sum_{n=2}^{\infty} a_{n} \\
\leq & \sum_{n=2}^{\infty}\left(\frac{(1+\beta \alpha) n}{\beta(2(k-2 \sigma)+\alpha)-(2 k-1)}+\frac{2(\beta(k-2 \sigma)-k)}{\beta(2(k-2 \sigma)+\alpha)-(2 k-1)}\right) a_{n},
\end{aligned}
$$

and as $f \in S_{c}^{\star} T(\alpha, \beta, \sigma, k)$, using the inequality in Theorem 2 yields

$$
\sum_{n=2}^{\infty} a_{n} \leq \frac{\beta(2(k-2 \sigma)+\alpha)-(2 k-1)}{2(1+\beta \alpha)+2(\beta(k-2 \sigma)-k)} .
$$

From (2) with $|z|=r(r<1)$, we have

$$
|f(z)| \leq r+\sum_{n=2}^{\infty} a_{n} r^{n} \leq r+\sum_{n=2}^{\infty} a_{n} r^{2}
$$

and

$$
|f(z)| \geq r-\sum_{n=2}^{\infty} a_{n} r^{n} \geq r-\sum_{n=2}^{\infty} a_{n} r^{2}
$$

Finally, using (9) in the above inequalities, give the result in Theorem 5.

The result in Theorem 5 is sharp for functions given by

$$
f_{2}(z)=z-\frac{\beta(2(k-2 \sigma)+\alpha)-(2 k-1)}{2(1+\beta \alpha)+2(\beta(k-2 \sigma)-k)} z^{2}
$$

at $z= \pm r$.

For completeness, we state the following result with regards to the class $S_{s c}^{\star} T(\alpha, \beta$, $\sigma, k)$.

Theorem 6. Let the functions $f$ be defined by (2) and belongs to the class $S_{s c}^{\star} T(\alpha, \beta$, $\sigma, k)$. Then for $\{z: 0<|z|=r<1\}$,

$$
r-\frac{\beta(2(k-2 \sigma)+\alpha)-(2 k-1)}{2(1+\beta \alpha)} r^{2} \leq|f(z)| \leq r+\frac{\beta(2(k-2 \sigma)+\alpha)-(2 k-1)}{2(1+\beta \alpha)} r^{2} .
$$

The result is sharp.

The result in Theorem 6 is sharp for functions given by

$$
f_{2}(z)=z-\frac{\beta(2(k-2 \sigma)+\alpha)-(2 k-1)}{2(1+\beta \alpha)} z^{2}
$$

at $z= \pm r$. 


\section{References}

[1] J. Clunie and F. R. Keogh, On starlike and Schlicht functions, J. London. Math. Soc. 35(1960), 229-233.

[2] R. M. El-Ashwa and D. K. Thomas, Some subclasses of close-to-convex functions, J. Ramanujan Math. Soc. 2(1987), 86-100.

[3] S. A. Halim, A. Janteng and M. Darus, Coefficient properties for classes with negative coefficients and starlike with respect to other points, 13th Mathematical Sciences National Symposium Proceeding (2005).

[4] K. Sakaguchi, On certain univalent mapping, J. Math. Soc. Japan. 11(1959), 72-75.

[5] H. Silverman, Univalent functions with negative coefficients, Proc. Amer. Math. Soc. 51(1975), 109-116.

Institute of Mathematical Sciences, Universiti Malaya, 50603 Kuala Lumpur, Malaysia.

E-mail: suzeini@um.edu.my

Institute of Mathematical Sciences, Universiti Malaya, 50603 Kuala Lumpur, Malaysia.

E-mail: aini_jg@ums.edu.my

School of Mathematical Sciences, Faculty of Sciences and Technology, Universiti Kebangsaan Malaysia, 43600 Bangi, Selangor, Malaysia.

E-mail: maslina@pkrisc.cc.ukm.my 